\title{
Enhanced Thinning Based Finger Print Recognition
}

\author{
${ }^{[1]}$ Parul Mishra, ${ }^{[2]}$ Ajit Kumar Shrivastava, ${ }^{[3]}$ Amit Saxena \\ ${ }^{[1]}$ Department of CSE, Truba Institute of Engg. and Information Technology, Bhopal, \\ M.P. , INDIA \\ Mishra.parul2009@gmail.com

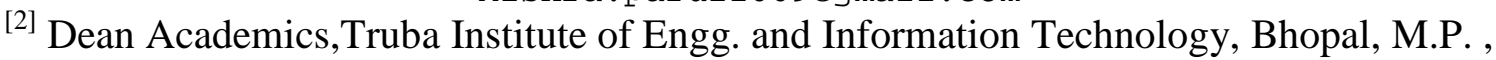 \\ INDIA \\ ajitshrivastavaerediffmail.com \\ ${ }^{[3]}$ Head of the Department, Truba Institute of Engg. and Information Technology, \\ Bhopal, M.P., INDIA \\ amitsaxena@trubainstitute.ac.in
}

\begin{abstract}
This paper is the implementation of fingerprint recognition system in which the matching is done using the Minutiae points. The methodology is the extracting \& applying matching procedure on the Minutiae points between the sample fingerprint \& fingerprint under question. The main functional blocks of this system follows steps of Image Thinning, Image Segmentation, Minutiae (feature) point Extraction, \& Minutiae point Matching. The procedure of Enhanced Thinning included for the purpose of decreasing the size of the memory space used by the fingerprint image database.
\end{abstract}

\section{Keywords}

Minutiae; Fingerprint; Segmentation; Image Thinning

\section{Introduction}

Fingerprint Recognition can be defined as the automated method of verifying a match between two human fingerprints. A finger print refers to the unique impression left by the friction ridges found on the inner surface of a finger or a thumb. In order to match two fingerprints the samples should be analysed and compared on the basis of some special features called Minutia points. There are two major minutia features that can be found on fingerprint ridges: Ridge Ending and Ridge Bifurcation. [1] A good quality fingerprint typically contains 40-100 minutiae [2, 3], as shown in the figure 1 .

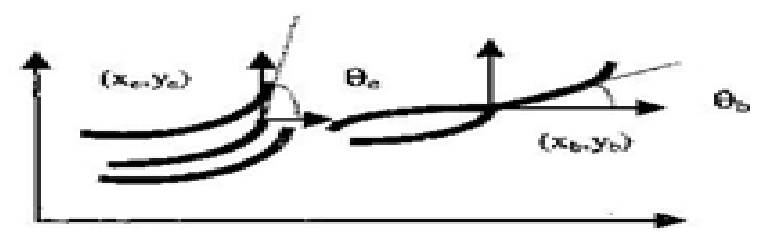

Figure 1 Finger Print

DOI: $10.5121 /$ ijci.2013.2204 
Fortunately, controlled, scientific testing initiatives are not limited within the biometrics community to fingerprint recognition. Other biometric modalities have been the target of excellent evaluation efforts as well. The (US) National Institute of Standards and Technology (NIST) has sponsored scientifically-controlled tests of text-independent speaker recognition algorithms for a number of years and, more recently, of facial recognition technologies as well.

In pattern recognition system, Feature Extraction (in general) is the process extracting information from the input which is useful for determining its category. In the case of fingerprints a natural choice are features based directly on the fingerprint ridges and ridge-valley structure. However, the effectiveness of a feature extraction can be determined by the image quality. Consequently, fingerprint image enhancement has become a necessary and common step after image acquisition and before feature extraction in most AFIS. Following, binarization, feature extraction and matching algorithms are executed on the enhanced image. The fingerprint enhancement can be employed on, both the gray-scale, and binary images, in spatial or frequency domain. In this paper we propose method based on Enhanced thinning of Images of original input gray-scale fingerprint image in frequency domain.

The rest of this paper progresses as follow. In Section II, Background and Literature survey presented and its characteristics in frequency domain are briefly described. In Section III, proposed methodology is explained. Parameters of proposed technique as well as results of enhancement obtained from available database sets are shown in Section IV, and conclusion is given in Section V.

\section{TABLE I}

\section{PRIMARY CONCERNS IN FINGERPRINT IMAGE RECOGNITION}

a) Clarity: It is very important for the image to be clear before extracting the minutiae points. Therefore Enhancement Techniques are used to obtain high and accurate match scores.

b) Noise Reduction: By calculating the ROI (Region of Interest), background information without effective ridges is eliminated.

c) Reduced Problem Space: A threshold is set on the basis of match scores for the application of Enhanced Thinning where noticeable increase in matching percentage can be seen.

d) Matching scores: Higher matching scores can be obtained by applying Enhanced Thinning.

\section{Background and Literature Survey}

Bana. S, with her colleague in 2011 presents a technique which is based on Minutiae based matching. This approach mainly depends on extraction of minutiae point from the sample finger print images and then performs matching based on the number of minutiae pairing among two fingerprints [4].

In 2009, Andelija M, et.al proposed an algorithm to enhance the fingerprint image based on adaptive filtering in frequency domain. Due to development of fast algorithms and power of modern computer systems, the filtering is often done in frequency domain. They propose 
two filter realizations for adaptive filtering in frequency domain, where both of them enhance fingerprint ridge-valley structure and attenuate existing noise [5].

In 2010, the proposed work for multi-scale and multi-directional recognition of fingerprints by K.Thaiyalnayaki, with his colleague included effective combination of features. Standard deviation, kurtosis, and skewness are the features which are included in their work. They apply the method by analyzing the finger prints with discrete wavelet transform (DWT) [6].

For Binary images a new parallel thinning algorithm was proposed by A. Jagna, in 2010 [7]. This proposed work was designed to solve the problem of excessive erosion and discontinuity in the images obtained after thinning in ZS and LW algorithm. The execution of this algorithm includes that the binary image undergoes two iterations of thinning known as two pass parallel thinning. This process preserves the end points and makes the image one pixel wide. The 8neighbour connectivity is also ensured by this algorithm. The reduction in end points is also observed here. [8-9]. However, the proposed algorithm shows the better performance and produces more quality images than the previous algorithms.

In 2005, Eun-Kyung Yun, Jin-Hyuk Hong and Sung-Bae proposed an adaptive pre-processing method, which extracts five features from the fingerprint images, analyses image quality with Ward's clustering algorithm, and enhances the images according to the characteristics. Fig.2 shows the overview of the proposed system in this paper. For fingerprint image quality analysis, it extracts several features in fingerprint images using orientation fields, at first. Clustering algorithm groups fingerprint images with the features, and the images in each cluster are analyzed and pre-processed adaptively [10].

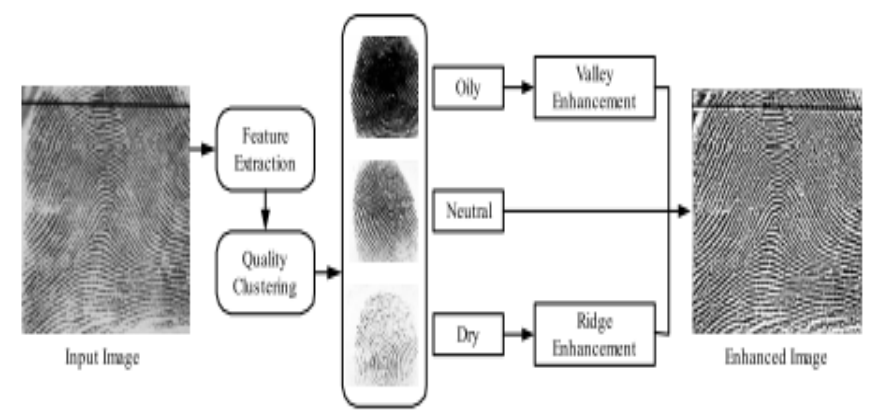

Figure 2 Adaptive Enhancing of Finger Print Images

Madhuri and Richa Mishra in 2012 propose a fingerprint recognition technique which uses local robust features for fingerprint representation and matching. The technique performs well in presence of rotation and able to carry out recognition in presence of partial fingerprints [11]. 


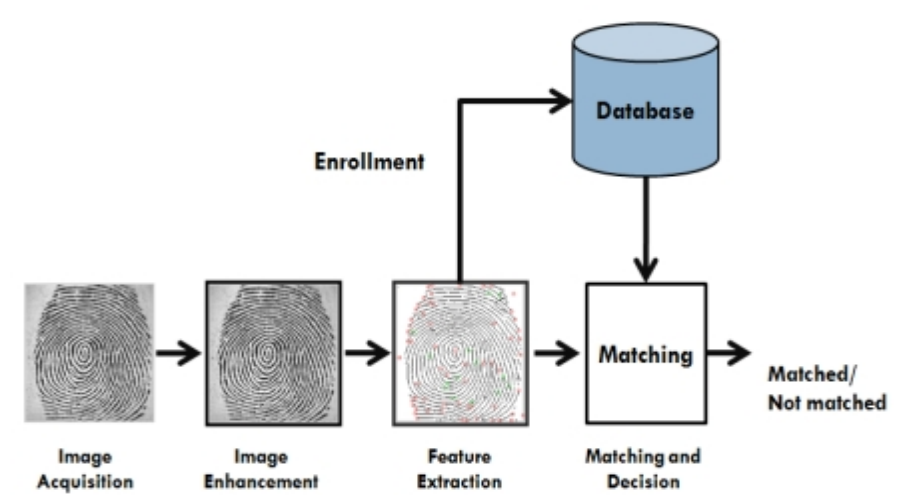

Fig 3. Finger Print based on Local robust features

\section{Finger Print Matching Techniques}

The Fingerprint Matching techniques can be broadly classified into three categories:

\section{Correlation-based matching:}

In this technique the correlation between two fingerprint images, for different displacements and rotations can be computed by superimposing them.

\section{Minutiae-based matching:}

It is the most widely used fingerprint matching technique. Here the minutiae are extracted from the two fingerprints and for increasing the efficiency of the matching process, the extracted minutiae points are stored in the matrix form. In this technique the template and the input minutiae sets are matched to generate the matching scores.

\section{Pattern-based (or image-based) matching:}

Pattern based algorithms compare the basic fingerprint patterns (arch, whorl, and loop) between a template and a candidate fingerprint. This requires that the images be aligned in the same orientation. This algorithm deals with finding a central point and centres on that. In a pattern-based algorithm, the template contains the size, type and orientation of patterns within the aligned fingerprint image. Then the graphical comparison is done between the candidate fingerprint and the template to determine the degree to which they match.

\section{Issues with Existing techniques}

Most of the existing fingerprint techniques in literature are based on minutiae points which are represented using their co-ordinate locations in the image. When test fingerprint image is rotated with respect to enrolled image or partially available, these techniques face problem in matching due to change in the co-ordinate locations of the minutiae points and perform very poorly. These two cases are discussed below. 


\section{Rotated Fingerprint Matching}

An example of a rotated fingerprint image is shown in Figure 3(b). We can see that it is difficult to match minutiae of two images because due to rotation, coordinate locations of all the minutiae points in Figure 3(b) with respect to Figure 3(a) are changed.
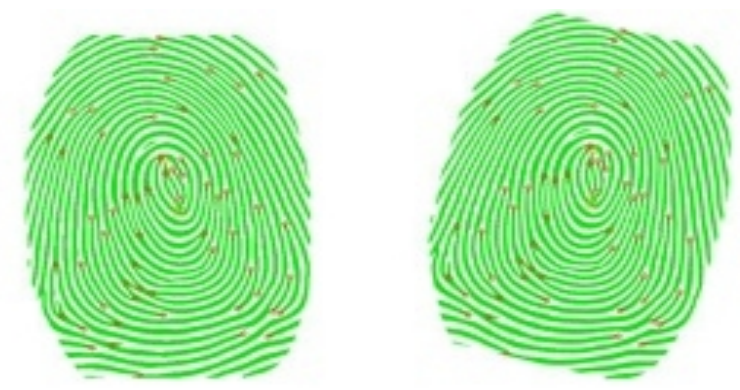

Figure 3 (a) Normal Fingerprint Image, (b) Rotated Fingerprint Image

\section{Partial Fingerprint Matching}

Figure 4(b) shows the example of partial fingerprint. We can see that it is difficult to match minutiae of two images because due to missing part of the fingerprint coordinate locations of all the minutiae points in Figure 4(b) with respect to Figure 4(a) are changed.

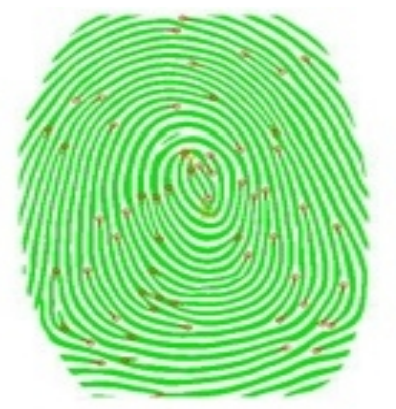

Figure 4 (a) Full Fingerprint

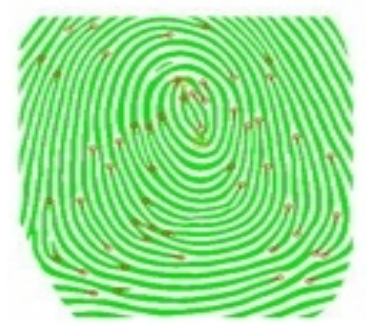

(b) Partial Fingerprint Image

\section{Proposed Methodology}

The Fig 5 shows the proposed methodology. Basic steps involves are written below:

\section{Image Enhancement}

Fingerprint Image Enhancement is the first step in the minutiae extraction process. For better performance of any fingerprint recognition system it is very important that the fingerprint images should be clear. With clear images higher matching scores can be obtained. As the fingerprint images are generally obtained from the scanner or other media therefore there is no guarantee of their good quality. The Image Enhancement techniques can be applied to increase the contrast between ridges and valleys and for connecting the false broken points of ridges. 
Originally, the enhancement step was supposed to be done using the canny edge detector. But after trial, it turns out that the result of an edge detector is an image with the borders of the ridges highlighted. For image enhancement we use:

\section{a. Histogram Equalization \\ b. Fast Fourier Transform}

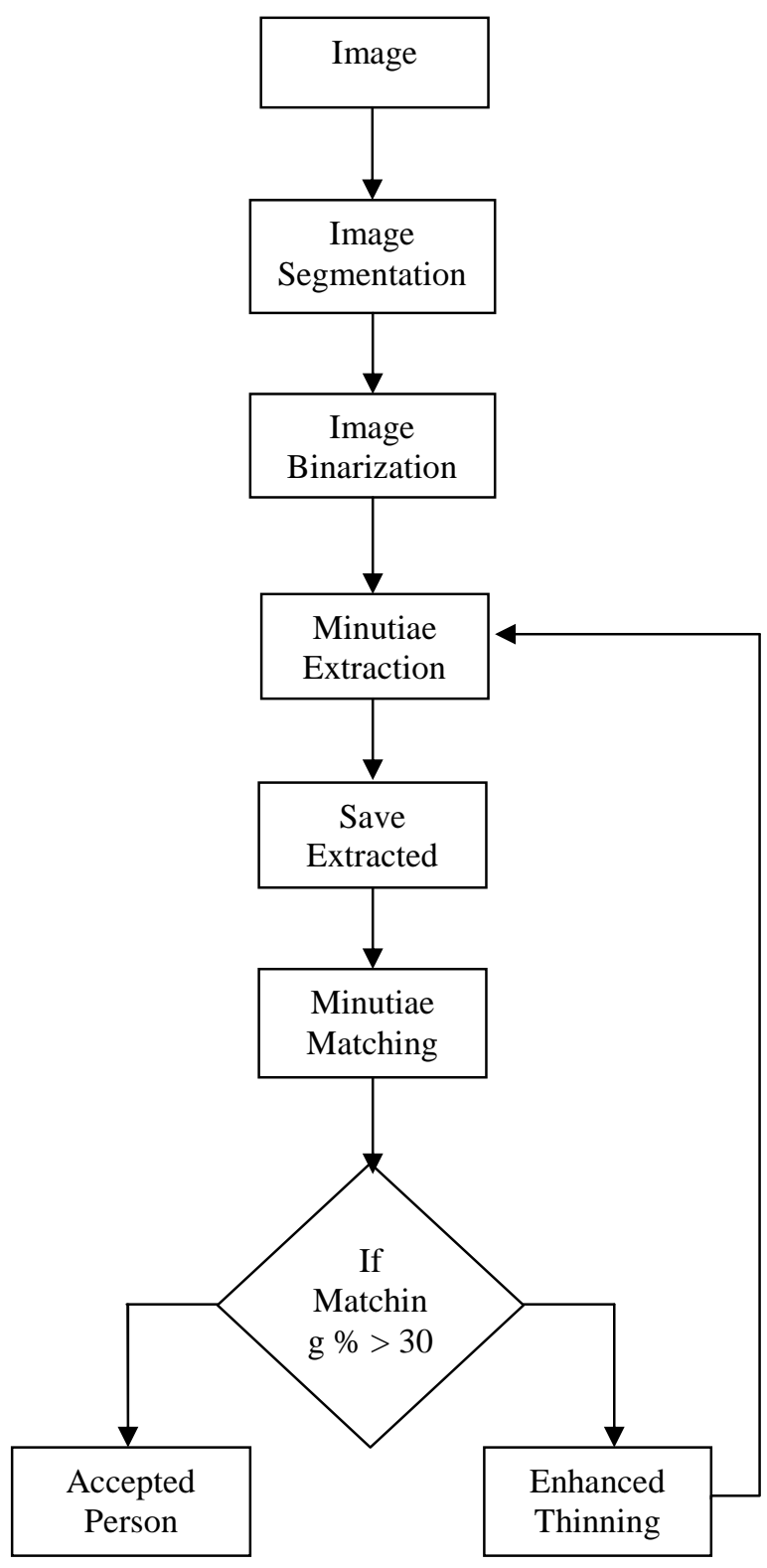

Figure 5 Flow chart of Proposed Algorithm 


\section{Image Binarization}

The binarization step is basically stating the obvious, which is that the true information that could be extracted from a print is simply binary; ridges vs. valleys. But it is a really important step in the process of ridge extracting, since the prints are taken as grayscale images, so ridges, knowing that they're in fact ridges, still vary in intensity. So, binarization transforms the image from a 256-level image to a 2-level image where the information remains same. Typically, an object pixel corresponds to a value of "1" while a background pixel corresponds to a value of " 0 ." Finally, depending on a pixel's value (black for 0 , white for 1), a binary image is obtained by colouring each pixel white or black.

\section{Image Segmentation}

Image Segmentation is the process of recognizing Region of Interest (ROI) for each fingerprint image. The area of the image without effective ridges is first discarded since it only holds background information or noise. Then the bound is sketched out for the remaining effective area in order to avoid confusion between the minutiae in the bound region with those false minutiae. The false minutiae are generated as a result of the ridges that are out of the sensor. There is a two-step method for extracting ROI. Used techniques are:

a) Block Direction Estimation

b) ROI extraction by Morphological Operation

\section{Final Minutiae Extraction}

Ridge Thinning is the process of eliminating the redundant pixels of ridges till the width of the ridges become just one pixel. An iterative, parallel thinning algorithm is used. The algorithm takes a small window of $(3 \times 3)$ in each scan and marks down the redundant pixels. And after several scans finally removes all those marked pixels. After this by using Morphological operations the thinned ridge map is filtered in order to remove some $\mathrm{H}$ breaks, isolated points and spikes. In this step, any single points, whether they are single-point ridges or single-point breaks in a ridge are eliminated and considered processing noise.

\section{Minutia Marking}

After Thinning of the fingerprint ridges, marking minutia points is relatively easy. For extraction of minutiae, the concept of Crossing Number $(\mathrm{CN})$ is widely used.

In a fingerprint image, for each $3 \times 3$ window, the central pixel is said to be a Ridge Branch, if its value is 1 and has exactly 3 one-value neighbours. [Figure 5.1]

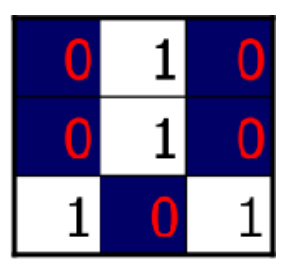

Figure 5.1 
And the central pixel is said to be a Ridge Ending if its value is 1 and has only 1 one-value neighbour. [Figure 5.2]. Therefore for any pixel $\mathrm{P}$, if $\mathrm{Cn}(\mathrm{P})==1$ it is called a Ridge End point and if $\mathrm{Cn}(\mathrm{P})==3$ it is called a Ridge Bifurcation point.

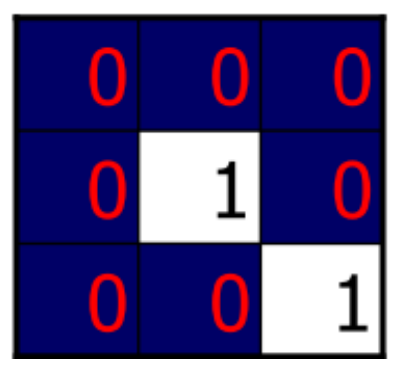

Figure 5.2

Fig 4.3 shown below illustrates a special case that a genuine branch is triple counted. If the uppermost pixel has value 1 and the rightmost pixel with value 1 , have another neighbour outside the $3 \times 3$ window, then the two pixels will be marked as branches but actually only one branch is located in the small region. So a check routine is added requiring that none of the neighbours of a branch are branches.

Now D is estimated which is the Average Inter-Ridge Width. The average inter-ridge width can be defined as the average distance between two neighbouring ridges. It is simple to approximate the value of D. Scan a row of the thinned ridge image and sums up all the pixels in the row with value one. Then divide the row length by the above summation to obtain the inter-ridge width. To provide more accuracy, several other rows and column scans are also conducted, finally all the inter-ridge widths are averaged to get the D.

In addition to marking the minutiae, all the thinned ridges in the fingerprint image are labelled with a unique ID for further operation

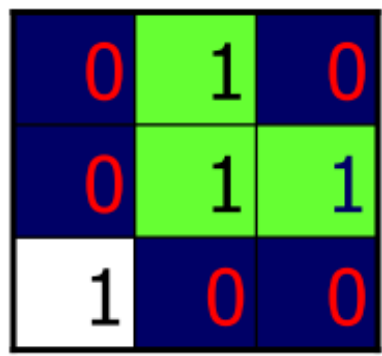

Figure 5.3

\section{Minutiae Post-Processing}

\section{False Minutia Removal}

There are some problems that cannot be totally fixed at the pre-processing stage. There are some problems that are not completely eliminated, caused due to insufficient amount of ink like false ridge breaks and ridge cross-connections due to over inking. Actually some artefacts are 
introduced at the earlier stages occasionally which later lead to spurious minutia. These false minutiae affects the accuracy of matching if they are simply regarded as genuine minutiae. So there should be some methods of removing false minutia which are essential to keep the fingerprint verification system effective.

\section{Enhanced Thinning}

Enhanced Thinning algorithm involves eight neighborhood. However, to preserve the connectivity is difficult here. Therefore to handle this problem, we use a 3 x 3 mask. The mask shown in Figure-6 (a \& b) indicating the eight neighboring pixels variations. A connectivity value is the sum of each weight in eight directions. After calculating the value of connectivity and applying specific conditions, decision can be made whether to delete the object pixel or to preserve. An essential point is defined as one which includes a connect point and an end point. The connect point is a point that its removal causes a disconnectivity in $3 \times 3$ mask. The end point can be defined as the point having only one of the eight-adjacent points. Proposed algorithm simply applies the above definitions so that the connectivity of the entire image can be maintained, to overcome the deficiencies of previous parallel thinning algorithms. The proposed algorithm consists of two steps i.e., Rule 1, In this step the value of connectivity for the entire image is calculated step by step and Rule 2 eliminates non-essential pixels step by step from the entire image. The pixel elimination process can be terminated if all the pixels are found essential. In Sequential Image Thinning algorithm the retention or deletion of a (black) pixel $\mathrm{p}$ depends upon the configuration of pixels in a local neighborhood containing $\mathrm{p}$, and the deletion of $\mathrm{p}$ in the nth iteration depends upon the operations undergone so far in the (n1)th iterations, and on the pixels that are processed in the nth iteration. In Proposed Parallel Image Thinning Algorithm, the elimination of pixels in the nth iteration would depends only on the output produced after the (n-l)th iteration, therefore, the processing of all the pixels can be takes place independently in a parallel manner, and thereby producing better quality thinned images, without excessive erosion and with 8-connectivity.

\begin{tabular}{|c|c|c|}
\hline $\mathrm{X}$ & $\mathrm{X}$ & $\mathrm{X}$ \\
\hline 1 & $\mathrm{Pi}$ & 1 \\
\hline $\mathrm{X}$ & 1 & $\mathrm{X}$ \\
\hline
\end{tabular}

Figure $6(a)$

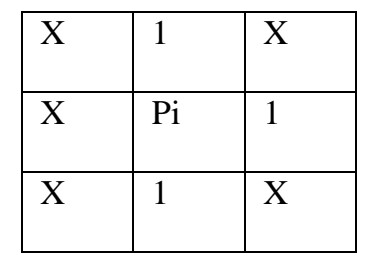

Figure 6 (b)

\section{Implementation and Result}

Implementation of proposed system requires better image quality, in order to produce match scores with higher accuracy. So concerning with this fact we develop a system which enhances the fingerprint image to provide clarity.

Background information and processing noise are the two major issues of this Fingerprint Recognition System. Following are the details of elimination of background information and processing noise in the proposed system. 
International Journal on Cybernetics \& Informatics ( IJCI) Vol.2, No.2, April2013

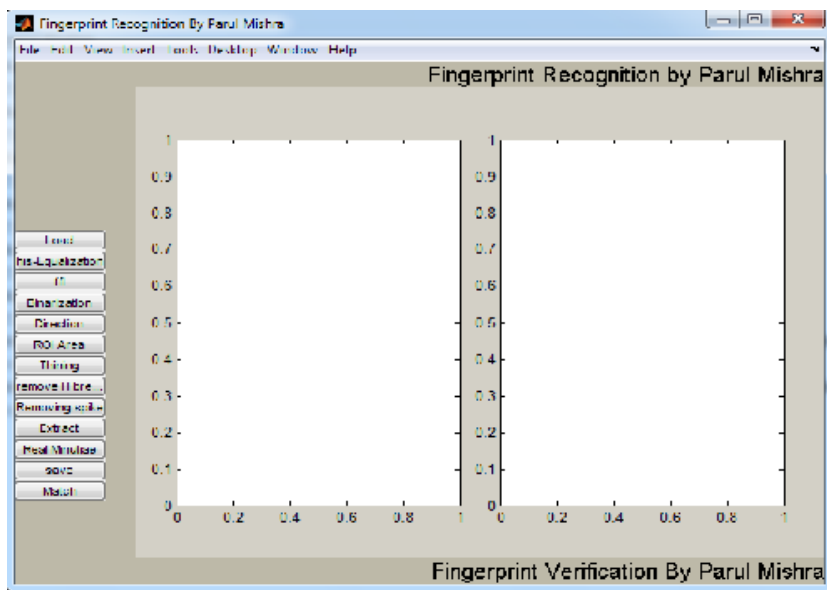

Figure 6.1 Main GUI of proposed Approach

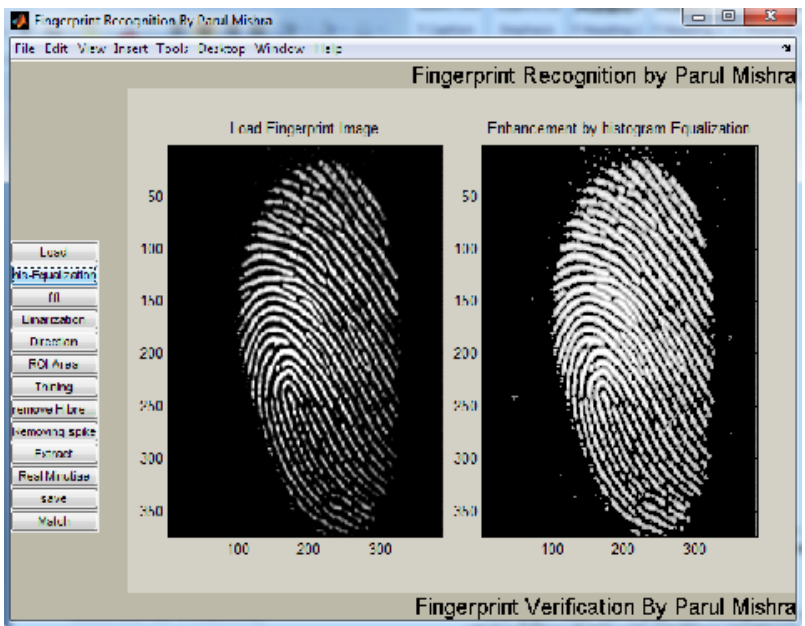

Figure 6.2 Histogram Equalized Image

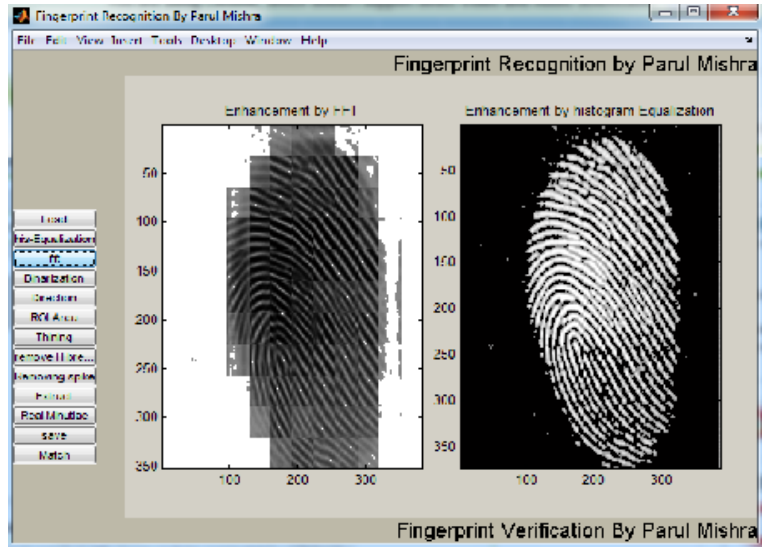

Figure 6.3 FFT Enhanced Image 
International Journal on Cybernetics \& Informatics ( IJCI) Vol.2, No.2, April2013

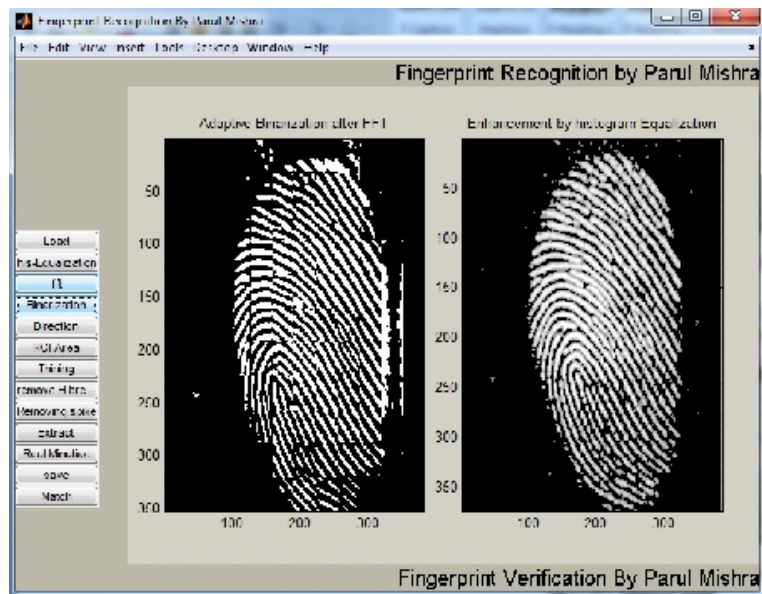

Figure 6.4 Binarized Image

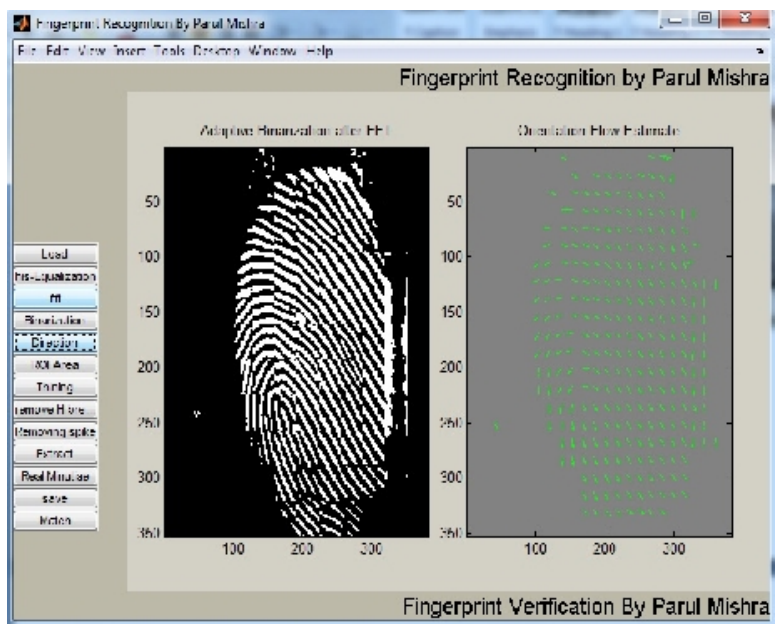

Figure 6.5 Direction Finded Image

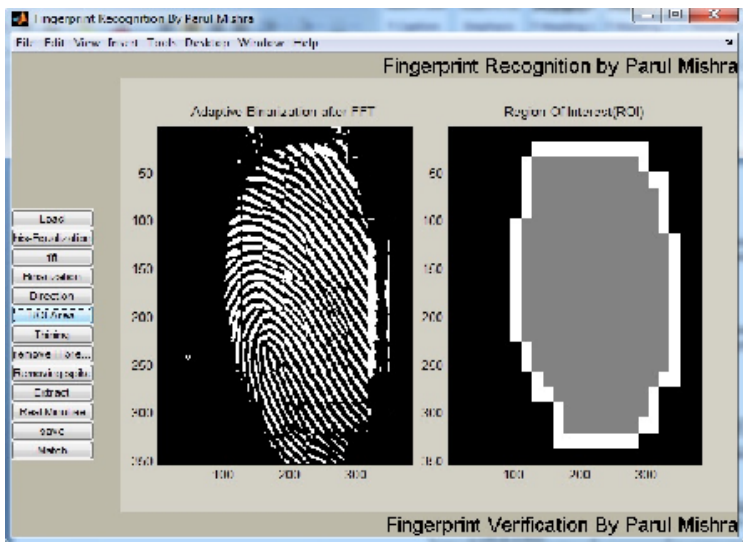

Figure 6.6 ROI extracted Image 
International Journal on Cybernetics \& Informatics ( IJCI) Vol.2, No.2, April2013

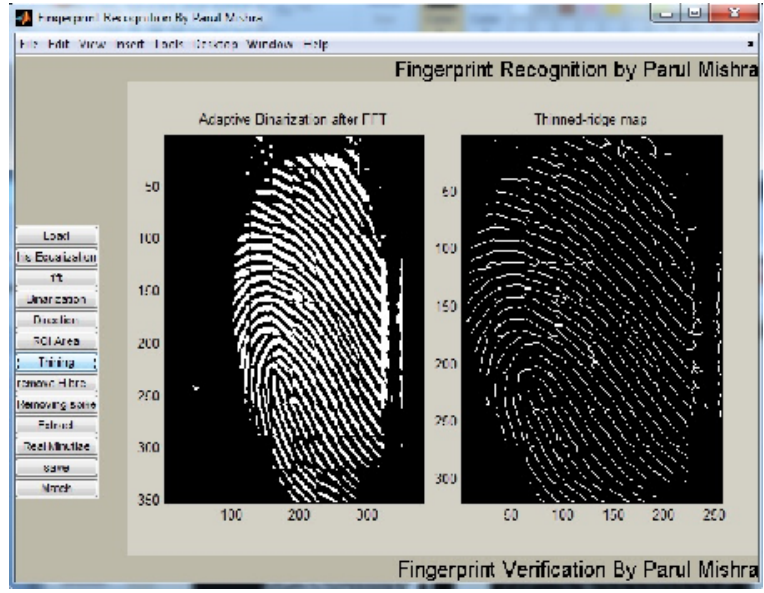

Figure 6.7 Thinned Image

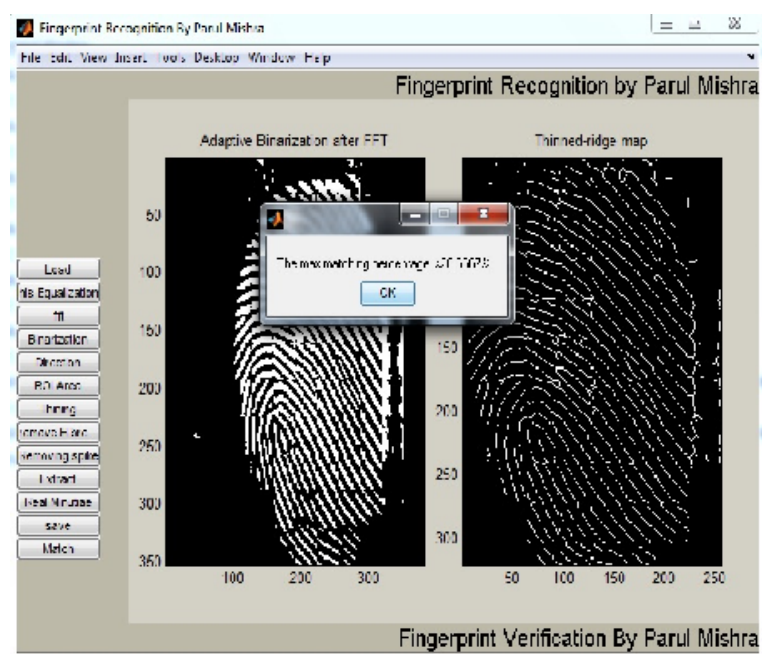

Figure 6.8 Case When Enhanced Thinning Required

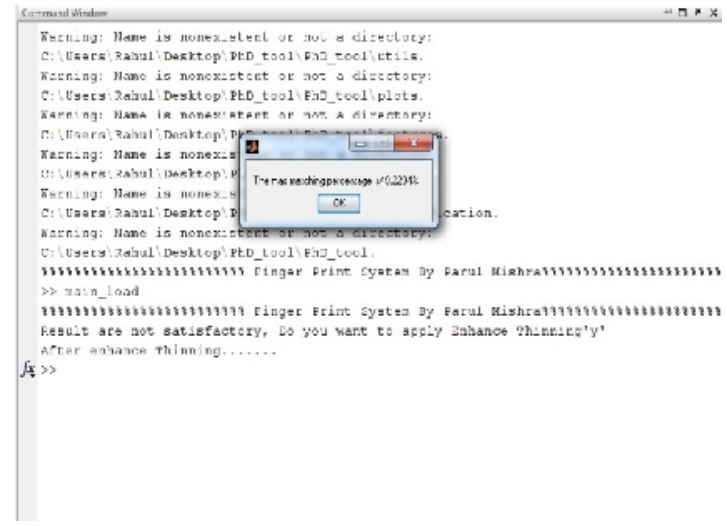

Figure 6.9 Results after Enhanced Thinning 


\section{TABLE II}

\section{COMPARISION OF RESULTS}

\begin{tabular}{|c|c|c|}
\hline Image & $\begin{array}{c}\text { Previous } \\
\text { Result }\end{array}$ & $\begin{array}{c}\text { Proposed } \\
\text { Result }\end{array}$ \\
\hline Img 1 & 26.66 & 49.24 \\
\hline Img 2 & 28.84 & 51.82 \\
\hline Img 3 & 24.56 & 48.56 \\
\hline Img 4 & 27.21 & 50.25 \\
\hline
\end{tabular}

\section{Discussions and Conclusion}

The process of Minutia Extraction plays a very important role in making any Automatic Fingerprint System reliable. Poor image quality is one of the most influential factors that can damage the process of locating minutia's correct location.

Enhanced Thinning based matching algorithm which is being proposed is found to be capable enough to locate the analogy or similarity between minutiae without adopting exhaustive research. Also the Enhanced Thinning algorithm is applied only to the images which are falling under a fixed threshold based on matching percentage so that the bad matching images can be separated from the good ones and thus the Enhanced thinning is applied only to the images under that threshold. After applying Enhanced Thinning to the images having low threshold, noticeable increase in the matching percentage is obtained.

The proposed work can also be improved by working on the accuracy and increasing the efficiency which can be achieved by applying the various image enhancement techniques or by the hardware improvements so that the captured images are more correct and valid. This will improve the input image to the thinning process that will directly reflect the better image quality

\section{References}

[1] "Minutiae Detection Algorithm for Fingerprint Recogenition”, IEEE AESS Systems Magazine, 2002.

[2] Jain A., Lin Hong \& Sharath Pankanti, "An Indentification System Using Fingerprints", IEEE Proceedings, Vol. 85, 1997.

[3] Jain A., Bolle R. \& Sharath Pankanti, " Biometric Peersonal Idetification in Networked Society", Kluwer Academic Publishers.

[4] S.Bana, D.Kaur, "Fingerprint Recognition using Image Segmentation", INTERNATIONAL JOURNAL OF ADVANCED ENGINEERING SCIENCES AND TECHNOLOGIES, Vol No. 5, Issue No. 1, $012-023$

[5] Andelija M, Brankica. M, “An Effective and Robust Fingerprint Enhancement by Adaptive Filtering in Frequency Domain”, ELEC. ENERG. vol. 22, no. 1, April 2009, 91-104.

[6] K.Thaiyalnayaki, S.S.A.Karim, P. V. Parmar, "Finger Print Recognition using Discrete Wavelet Transform", International Journal of Computer Applications (0975 - 8887), Volume 1 - No. 24, 2010.

[7] A. Jagna, V. Kamakshiprasad, "NEW PARALLEL BINARY IMAGE THINNING ALGORITHM", ARPN Journal of Engineering and Applied Sciences, VOL. 5, NO. 4, APRIL 2010 
International Journal on Cybernetics \& Informatics ( IJCI) Vol.2, No.2, April2013

[8] T .Y. Zhang and C.Y. Suen. 1984. A Fast Parallel Algorithms for Thinning Digital Patterns. Research Contributions, Communications of the ACM. 27(3): 236-239.

[9] H.E. Lu and P.S.P. Wang. 1985. An improved fast parallel algorithm for thinning digital patterns. Proc. of the IEEE Conf. on computer vision and pattern recognition. pp. 364-367.

[10] Eun-Kyung Yun, Jin-Hyuk Hong and Sung-Bae Cho, "Adaptive Enhancing of Fingerprint Image with Image Characteristics Analysis", AI 2004: Advances in Artificial Intelligence, Lecture Notes in Computer Science Volume 3339, 2005, pp 120-131

[11] M. Mishra, R. Mishra, "Fingerprint Recognition using Robust Local Features", International Journal of Advanced Research in Computer Science and Software Engineering, Volume 2, Issue 6, June 2012 ISSN: 2277 128X.

\section{Authors:}

Mr. Ajit Kumar Shrivastava working as the Dean Academics of Truba Institute of Engineering \& Information Technology, Bhopal. He is having a vast experience in the field of Computer Science and Academics. He has guided a number of Projects and Dissertations for M. Tech. Students, as well as for UG Students. He has completed his Engineering (Honors) in Computer Technology, Master of Engineering (Honors) in Computer Engineering.

Mr. Amit Saxena working as the Head of the Department of Computer Science \& Engineering in Truba Institute of Engineering \& Information Technology, Bhopal. He is having Nine years of experience in the field of Computer Science and Academics. He has guided more than 40 Projects and Dissertations for M. Tech. Students, as well as for UG Students. He has completed his Engineering (Honors) in Computer Science \& Engineering, Master of Engineering (Honors) in Computer Science Engineering. His area of research is Security Issues in Mobile Adhoc Network and Behavioral Analysis of Selfish and Malicious Nodes in MANET.

Parul Mishra has done her Engineering from Shree Institute of Science \& Technology, Bhopal (RGPV) in Computer Science Engineering Branch. She is having an Experience of 1 Year in Academics, worked as an Assistant Professor (CS/IT) in People's College of Research \& Technology, Bhopal. Currently, she is pursuing M.Tech. (Final Semester) in Computer Science Engineering Branch from Truba Institute of Engineering \& Information Technology, Bhopal (RGPV). Her areas of interest are Security, Object Oriented Programming, Operating Systems, Data Base Management Systems, etc. Her topic for research is Finger Print Recognition. 